\title{
Medidas auto-referidas são válidas para avaliação do estado nutricional na população brasileira?
}

\author{
Are self-reported measures valid for the assessment \\ of nutritional status in the brazilian population?
}

\author{
Raildo da Silva Coqueiro ${ }^{1,3}$ \\ Lucélia Justino Borges 1,4 \\ Valbério Candido Araújo 2,4 \\ Andreia Pelegrini $\quad$ 1,4 \\ Aline Rodrigues Barbosa
}

1 Universidade Federal de Santa Catarina. Programa de Pós-Graduação em Educação Física. Grupo de Pesquisa de Cineantro pometria e Desempenho Humano. Florianópolis, SC. Brasil.

2 Universidade Federal de Santa Catarina. Programa de Pós-Graduação em Educação Física. Núcleo de Pesquisa em Atividade Física e Saúde. Florianópolis, SC. Brasil.

3 Bolsista CNPq

4 Bolsista Capes

Recebido em 10/04/08 Aprovado em 01/08/08
Resumo - As medidas auto-referidas têm sido amplamente utilizadas para avaliar o estado nutricional em estudos epidemiológicos. Este ponto de vista teve o propósito de tecer considerações sobre a validade do uso das medidas de massa corporal e estatura referidas, para a avaliação do estado nutricional em crianças, adolescentes, adultos e idosos no Brasil. As evidências disponíveis sugerem que: a) em crianças, a utilização de medidas auto-referidas não é recomendada, devido à inexistência de estudos nacionais; b) em adolescentes, essas medidas devem ser utilizadas com cautela, visto que o único estudo realizado no país foi restrito a uma amostra local e pode não representar a realidade das demais regiões; c) em adultos, o uso desse método pode ser considerado uma alternativa viável para avaliar e monitorar o estado nutricional, em situações nas quais não é possível a medida direta dessas informações; d) em idosos, ainda não é possível recomendar a utilização das medidas auto-referidas por dois motivos: 1) os estudos não foram conduzidos especificamente com indivíduos acima de 60 anos; 2) esse grupo etário tende a estimar com menor acurácia essas medidas.

Palavras-chave: Antropometria; Confiabilidade e validade; Estado nutricional; Índice de massa corporal; Pesos e medidas corporais.

Abstract - Self-reported measures have been widely used for the assessment of nutritional status in epidemiological studies. This paper discusses aspects regarding the validity of self-reported measures of body weight and height for the assessment of nutritional status in Brazilian children, adolescents, adults and elderly subjects. The available evidence suggests that: a) in children, the use of self-reported measures is not recommended because of the lack of Brazilian studies; b) in adolescents, these measures should be used with caution since the only study carried out in Brazil was restricted to a local sample and may not represent the situation in other regions; c) in adults, this method can be considered a viable alternative for assessing and monitoring nutritional status in situations in which these data cannot be measured directly; d) in the elderly, the use of self-reported measures cannot yet be recommended for two reasons: first, studies specifically including subjects older than 60 years have not been conducted and, second, this age group tends to estimate these measures less accurately.

Key words: Anthropometry; Reproducibility of results; Nutritional status; Body mass index; Body weight and measures. 


\section{INTRODUÇÃO}

O estado nutricional é uma condição de saúde que reflete os processos de ingestão, digestão, absorção, transporte, metabolismo, estoque e por fim, a excreção dos componentes alimentares e seus metabólitos¹. Ou seja, o tipo e a quantidade dos alimentos ingeridos, as necessidades energéticas do indivíduo e a eficiência do aproveitamento biológico dos nutrientes devem estar em equilíbrio. Sempre que algum fator interfere em qualquer etapa desse processo, os riscos de inadequação nutricional podem ocorrer.

Por isso, a avaliação do estado nutricional é um dos principais fatores a considerar em pacientes hospitalizados ${ }^{2}$, na monitoração da eficácia de intervenções dietoterápicas ${ }^{3}$ e na análise da adequação nutricional de uma população ou grupo de indivíduos ${ }^{4-6}$.

A avaliação do estado nutricional pode ser feita por medidas bioquímicas, clínicas, dietéticas, antropométricas e também por medidas da composição corporal (densitometria computadorizada, hidrodensitometria, isótopos marcados pela medição da água corporal total, análise da ativação de nêutrons in vivo e ressonância magnética) ${ }^{3}$. A antropometria (massa corporal, estatura, circunferências e dobras cutâneas) é o método mais utilizado por apresentar baixo custo, ser seguro, não invasivo e de relativa facilidade de execução.

As informações da massa corporal e da estatura verificadas, preferencialmente, por meio da mensuração direta, são necessárias para obtenção do índice de massa corporal [IMC = peso $(\mathrm{kg}) /$ estatura $\left.(\mathrm{m})^{2}\right]$, o indicador nutricional mais utilizado em estudos clínicos e populacionais ${ }^{2,4-6}$. Além do IMC, em adolescentes, a avaliação nutricional é feita através do indicador estatura/ idade e, em crianças, pelos indicadores peso/ idade, estatura/idade, peso/estatura, sendo o diagnóstico nutricional feito por percentil ou escore $\mathrm{Z}$ de acordo com a idade?

Embora as aferições da massa corporal e da estatura sejam realizadas de forma rápida, prática e com equipamentos de baixo custo, existem situações que podem justificar o uso de medidas referidas (indiretas), como na vigilância nutricional e epidemiológica ${ }^{6}$, podendo as informações ser obtidas por telefone, carta registrada, correspondência eletrônica, sem a necessidade do pesquisador em campo. $\mathrm{O}$ que faz diminuir as despesas e atingir locais, muitas vezes, de difícil acesso.
A validade das medidas referidas em diferentes grupos etários ainda é controversa. Os estu$\operatorname{dos}^{8-11}$ mostram que determinados fatores estão relacionados às subestimativa/ superestimativa da massa corporal e da estatura, podendo repercutir em erro quanto à freqüência de indivíduos com inadequação nutricional.

Portanto, este estudo teve o propósito de tecer considerações sobre a validade do uso das medidas de massa corporal e estatura referidas, para a avaliação do estado nutricional em crianças, adolescentes, adultos e idosos no Brasil.

\section{Validade em Crianças (A partir de 7 anos e menor que 10 anos)}

Não foram encontrados, na literatura nacional, estudos que tenham utilizado medidas referidas (massa corporal e estatura) em crianças e que, portanto, comprovem a confiabilidade dessas neste grupo etário. Internacionalmente, a validade do uso das medidas de massa corporal e estatura auto-referidas foram investigadas ${ }^{12,13}$ com a finalidade de verificar a aplicabilidade destas em pesquisas epidemiológicas e/ou clíni$\mathrm{cas}^{13}$. Foram encontrados apenas dois estudos, um realizado com escolares gregos $(n=378)^{12} \mathrm{e}$ outro com americanos $(\mathrm{n}=77)^{13}$.

No estudo realizado na Grécia, a prevalência de sobrepeso aferida e auto-relatada foi de $29 \%$ e $23 \%$, respectivamente, e a obesidade foi de $9 \%$ e $4 \%$, sendo encontrada alta correlação entre os índices antropométricos auto-referidos e aferidos ${ }^{12}$. Estes resultados indicaram subestimativa de sobrepeso em $6 \%$ e de obesidade em $5 \%$. As evidências encontradas nesse estudo indicaram discordâncias entre os métodos, as quais podem conduzir a estimativas erradas do estado nutricional em crianças. Portanto, os autores concluíram que, embora os dados auto-referidos sejam de fácil obtenção, medidas diretas são necessárias para a avaliação do sobrepeso e obesidade infantil.

No estudo americano, realizado em Boston, meninos e meninas subestimaram, significativamente, a massa corporal e a estatura, apesar do coeficiente de correlação intra-classe (CCI) mostrar boa ou excelente reprodutibilidade ${ }^{13}$.

Os resultados dos estudos internacionais sugerem que, as medidas auto-referidas não são válidas para a avaliação física de crianças obesas $^{13}$. No caso do Brasil, até que se realizem pesquisas nacionais em crianças, com amostras representativas ou sub-amostra, a utilização 
de medidas auto-referidas para a avaliação do estado nutricional não é recomendada.

A falta de pesquisas não permite identificar os possíveis fatores que podem influenciar o auto-relato da massa corporal e estatura das crianças brasileiras. Contudo, é possível especular que as diferenças socioeconômicas existentes no País podem sinalizar para o fato de que a freqüência, com que as crianças têm seu crescimento/estado nutricional verificado, não seja a mesma nas várias regiões. A escola, que poderia também realizar estas medidas rotineiramente, geralmente não o faz, por falta de material, tempo e/ou interesse; associado ao fato de que nem todas as crianças brasileiras estão matriculadas e/ou freqüentam o ambiente escolar. Acredita-se, também, que a preocupação com essas medidas ainda não faz parte do universo de grande parte das crianças brasileiras e que algumas teriam dificuldade de recordar a sua massa corporal e a sua estatura, fato que poderia, em pesquisas, interferir nos resultados dessas medidas.

\section{Validade em Adolescentes (A partir de 10} anos e menor que 20 anos)

Foi encontrado, na literatura nacional, apenas um estudo utilizando medidas de massa corporal e estatura auto-referidas ${ }^{11}$. A pesquisa foi conduzida em Florianópolis-SC, com amostra representativa de escolares de 15 a 18 anos (n $=867$ ). Os resultados mostraram subestimativa da massa corporal e superestimativa da estatura, resultando em subestimação do IMC de $0,07 \mathrm{~kg} / \mathrm{m}^{2}$ e $0,56 \mathrm{~kg} / \mathrm{m}^{2}$, para rapazes e moças, respectivamente. As medidas aferidas e autoreferidas apresentaram alto CCI e a diferença na prevalência de sobrepeso/obesidade entre os métodos não foi significativa. Entretanto, quando as proporções foram verificadas, de acordo com o sexo e idade, observou-se que somente as moças de 16 e 18 anos apresentaram diferenças significativas na prevalência de excesso de peso, com subestimação de 6,5\% e 7,0\%, respectivamente. Dessa forma, o autor concluiu que as medidas auto-referidas (massa corporal e estatura) são válidas como formas de aproximação aos valores mensurados, podendo ser utilizadas na avaliação do estado nutricional de adolescentes em estudos epidemiológicos, sendo que o único fator que influenciou a magnitude do erro no IMC foi o sexo, com subestimação maior nas moças.
De forma geral, os estudos internacionais ${ }^{14-16}$ não demonstraram boa acurácia das medidas auto-referidas para classificação do estado nutricional de adolescentes e sugeriram que esse método não deve ser empregado ${ }^{14,16} \mathrm{e}$ se for utilizado, que seja com cautela ${ }^{15}$.

De acordo com Gálan et al. ${ }^{14} \mathrm{e}$ Wang et al. ${ }^{15}$, o rápido incremento da massa corporal e estatura são fatores que podem contribuir para a menor acurácia do IMC calculado a partir de medidas auto-referidas ${ }^{14,15}$. Além disso, a preocupação com a imagem corporal tende a levar os adolescentes a relatarem a massa corporal e estatura com base em medidas que gostariam de ter, o que parece ser reforçado pelos elevados índices de insatisfação com a imagem corpora ${ }^{17}$.

Apesar de o único estudo nacional apresentar boa validade para o IMC, calculado por meio de medidas referidas, e sugerir a utilização desse método para o diagnóstico do estado nutricional de adolescentes em pesquisas epidemiológicas, é necessário destacar que os resultados mostraram diferenças importantes na prevalência de sobrepeso/obesidade em indivíduos do sexo feminino em alguns grupos etários (16 e 18 anos). Portanto, a vigilância nutricional e/ou epidemiológica por medidas referidas deve ser feita com cautela.

Ressalta-se, ainda, a necessidade de realização de estudos em outras localidades do país, visto que os resultados encontrados são derivados de uma amostra local e podem não representar a realidade das diversas regiões brasileiras.

\section{Validade em Adultos (A partir de 20 anos e menor que 60 anos)}

Para adultos, ao contrário do observado em relação às crianças e adolescentes, foram encontrados alguns estudos nacionais referentes à validade das medidas auto-referidas ${ }^{8-10,18}$, para identificação do estado nutricional (IMC), embora nem todos envolvam amostra representativa ou subamostra ${ }^{18}$ da população estudada.

Em pesquisa realizada na cidade de Petrópolis-RJ, envolvendo 328 indivíduos (18 a 81 anos), selecionados por conveniência, não foram encontradas diferenças significativas para ambos os sexos, entre os valores médios de massa corporal e estatura auto-referidas e aferidas ${ }^{18}$. Como conseqüência, as diferenças no IMC, calculado por ambos os métodos, também não diferiram. 
Foram encontrados CCI elevados, em homens e mulheres, entre os valores de IMC verificados pelos dois métodos. Os autores fomentam a utilização desse método em estudos de elevado número amostral. Entretanto, cabe ressaltar que a amostra do estudo não foi representativa e a prevalência de inadequação nutricional (baixo peso e sobrepeso) não foi comparada entre os dois métodos ${ }^{18}$.

Fonseca et al..$^{10}$ realizaram pesquisa com intuito de verificar a validade de peso e estatura informados e IMC em 3.713 funcionários públi$\cos (22$ a 70 anos) de uma universidade do Rio de Janeiro. O estudo mostrou que indivíduos de ambos os sexos subestimaram a massa corporal e superestimaram a estatura, resultando em subestimação do IMC auto-referido de $0,37 \mathrm{~kg} /$ $\mathrm{m}^{2}$ e $0,77 \mathrm{~kg} / \mathrm{m}^{2}$ para homens e mulheres, respectivamente. As prevalências de inadequação nutricional, segundo os dois métodos, não foram comparadas, porém, foram obtidos valores altos de sensibilidade e especificidade para determinar sobrepeso e obesidade. Os autores ${ }^{10}$ concluíram que medidas auto-referidas apresentaram boa concordância e validade, podendo ser utilizadas em populações semelhantes, quando a economia desses recursos for importante para a realização do estudo.

O estudo de base populacional realizado em Pelotas-RS, por Silveira et al. ${ }^{9}$, envolvendo uma sub-amostra de 131 pessoas, evidenciou que homens e mulheres tenderam a superestimar a estatura e a massa corporal. Porém, como os homens superestimaram a massa corporal e as mulheres a estatura, o resultado final resultou em superestimativa no IMC dos homens de $0,17 \mathrm{~kg} / \mathrm{m}^{2}$ e subestimativa de $0,99 \mathrm{~kg} / \mathrm{m}^{2}$ nas mulheres. Apesar dessas diferenças, foi observada alta concordância (Kappa e CCI) em ambos os grupos. No sexo masculino, as prevalências de obesidade calculadas pelo IMC auto-referido e aferido foram iguais (11\%) e as de sobrepeso se aproximaram ( $41 \%$ e 46\%, respectivamente). Já no feminino, a freqüência de obesidade foi de 18\% (referida) e 22\% (medida direta). É importante destacar que, quando o IMC foi corrigido, por meio de modelos de regressão múltiplos, a prevalência de obesidade referida para as mulheres foi de $22 \%$, ou seja, igual ao valor aferido. Os autores sugeriram que medidas indiretas podem ser efetivas para o monitoramento da prevalência de obesidade, embora devam ser utilizadas com cautela, pois pode haver necessidade de corre- ções, em determinados estratos, para a obtenção de estimativas mais confiáveis.

Peixoto et al. ${ }^{8}$, em pesquisa de base populacional, realizada em Goiânia-GO, mostraram que homens e mulheres superestimaram a estatura, o que contribuiu para a subestimação do $\operatorname{IMC}\left(0,27 \mathrm{~kg} / \mathrm{m}^{2}\right.$ e $0,67 \mathrm{~kg} / \mathrm{m}^{2}$, respectivamente), embora os valores de massa corporal aferida e referida, em ambos os sexos, não tenham apresentado diferenças significativas. No estudo ${ }^{8}$, foi encontrada alta concordância (CCI) entre o IMC calculado a partir das medidas diretas e indiretas. Além disso, as prevalências de baixo peso, eutrofia, sobrepeso e obesidade, de acordo com os dois métodos, não diferiram significativamente. Os autores concluíram que, para essa população, a massa corporal e a estatura, autoreferidas, constituem informações confiáveis para estudos epidemiológicos de monitoramento da prevalência de obesidade.

Todas as pesquisas ${ }^{8-10,18}$ relataram boa concordância e validade do IMC calculado por meio de medidas auto-referidas quando comparadas às aferidas. De acordo com essas pesquisas, diversos fatores podem afetar a validade do IMC, baseado em medidas de estatura e massa corporal referidas. Dentre os fatores associados à menor acurácia, destaca-se o sexo feminino $o^{8,9}$, obesidade ${ }^{8-10}$, idade mais avança$\mathrm{da}^{8-10}$, menor renda familiar ${ }^{9}$, menor estatura ${ }^{8} \mathrm{e}$ baixa escolaridade ${ }^{8}$.

O uso de medidas indiretas pode ser uma alternativa viável para avaliar e monitorar o estado nutricional em adultos brasileiros, em situações em que não é possível a mensuração direta dessas informações (inquéritos por telefone, carta registrada ou e-mail). Todavia, recomenda-se a utilização de uma sub-amostra que permita identificar os grupos de indivíduos mais susceptíveis a erros que podem comprometer a classificação do estado nutricional. A partir da identificação desses grupos será possível realizar correções (equações de regressão) que melhorem a acurácia das medidas referidas. Destaca-se, ainda, que devido às diversidades socioculturais do país, em regiões onde ainda não existem estudos de validação (Norte e Nordeste), a utilização de medidas auto-referidas na identificação do estado nutricional deve ser feita com cautela.

\section{Validade em Idosos (A partir de 60 anos)}

$\mathrm{Na}$ literatura nacional, não foram encontrados estudos com amostra representativa de idosos 
que utilizassem medidas auto-referidas da massa corporal e estatura, para identificação do estado nutricional. Algumas pesquisas populacionais, realizadas com adultos ${ }^{8,10}$, incluíram indivíduos de 60 anos e mais e mostraram que a acurácia das medidas indiretas tende a ser menor que em adultos.

O estudo de base populacional, realizado em Goiânia-GO, por Peixoto et al. ${ }^{8}$, com indivíduos de 20 a 64 anos, mostrou que homens e mulheres, do grupo etário 55 a 64 anos, superestimaram a estatura e apenas o sexo masculino subestimou a massa corporal. Esses achados resultaram na subestimação do IMC de 0,44 kg/m² e 1,18 kg/ $\mathrm{m}^{2}$ para homens e mulheres, respectivamente. Mesmo sendo detectada subestimação, os autores observaram alta concordância (CCI) entre o IMC aferido e o referido. Entretanto, como a comparação entre as prevalências de sobrepeso e obesidade foi verificada apenas para a amostra total do estudo (20 a 64 anos), não foi possível identificar se o IMC, obtido por meio de medidas auto-referidas, é um método válido para a avaliação do estado nutricional em idosos.

A pesquisa de Fonseca et al. ${ }^{10}$, realizada no Rio de Janeiro, com indivíduos de 22 a 70 anos, apresentou resultados para diferentes grupos etários. Restringindo-se às pessoas de 50 a 70 anos, verificou-se alta correlação (CCI) entre os valores aferidos e auto-referidos de massa corporal, estatura e IMC. Foram demonstrados, ainda, elevados valores de sensibilidade e especificidade para o IMC auto-referido no diagnóstico de sobrepeso e obesidade em ambos os sexos. Não foram comparadas as prevalências de sobrepeso e obesidade entre os dois métodos, todavia, os valores de sensibilidade e especificidade sugeriram boa validade das medidas auto-referidas. Os autores afirmam que o fato de incluir na amostra somente pessoas empregadas e selecionadas por concurso público, indica que a informação obtida, por meio desse método, pode ser de boa qualidade somente em populações similares, ou seja, em pessoas que possuem maior nível de escolaridade e trabalhadores de áreas urbanas.

Os achados dos estudos nacionais concordam com os observados em pesquisas internacionais que evidenciaram subestimação do IMC por indivíduos de ambos os $\operatorname{sexos}^{19} \mathrm{e}$ alta correlação entre as medidas aferidas e referidas $^{20}$. Além disso, as pesquisas internacionais apontam que os idosos estimam estatura e/ ou massa corporal com menor acurácia que as demais populações ${ }^{21,22}$ e os autores recomendam, exceto em nível individual ou na prática clínica $^{23}$, a utilização de medidas auto-referidas em pesquisas epidemiológicas de prevalência e monitoramento do estado nutricional de idosos $^{19,20}$.

De acordo com os estudos, a idade ${ }^{19,24}$, a condição socioeconômica ${ }^{24}$, a perda gradual da estatura com o envelhecimento ${ }^{19,24}$, a ausência de aferição freqüente dessas medidas ${ }^{21}$ e a baixa escolaridade são fatores que podem exercer forte influência na qualidade da informação auto-referida ${ }^{8}$, diminuindo a precisão dessas medidas.

No Brasil, apesar de alguns estudos terem demonstrado boa concordância entre as medidas aferidas e referidas, ainda não é possível recomendar a utilização desse método, visto que não foram encontradas pesquisas realizadas em amostras representativas da população idosa. Investigações envolvendo amostra probabilística devem ser conduzidas com esse grupo etário, a fim de verificar a validade das medidas autoreferidas e os fatores que podem influenciar a acurácia desse método, levando em consideração o baixo grau de instrução, a condição socioeconômica, as diferenças regionais e os fatores culturais tão fortes e presentes no país.

\section{CONSIDERAÇÕES FINAIS}

São vários os fatores que podem interferir na confiabilidade dos valores de medidas de massa corporal e estatura auto-referidas, e estes são específicos ao grupo etário analisado.

Acredita-se que, em crianças, a não realização dessas medidas regularmente e a provável falta de interesse delas pelo assunto, podem ser fatores que influenciam diretamente a acurácia das medidas. O rápido aumento da massa corporal e estatura e a preocupação com a imagem corporal podem contribuir para a menor confiabilidade do IMC em adolescentes. A menor acurácia das medidas auto-referidas em adultos está associada ao sexo feminino, à obesidade, à idade mais avançada, ao baixo nível socioeconômico, à menor estatura e à baixa escolaridade. Nos idosos, a idade avançada, a baixa escolaridade, a menor condição econômica, a perda gradual da estatura ocorrida com o envelhecimento e a ausência de aferição freqüente, são fatores relacionados à menor confiabilidade dos valores auto-referidos. 
Ao considerar a literatura disponível, é possível concluir que:

1) Em crianças, a utilização de medidas autoreferidas não é recomendada, devido à inexistência de estudos nacionais;

2) Em adolescentes, essas medidas devem ser utilizadas com cautela, visto que o único estudo realizado no país foi restrito a uma amostra local e pode não representar a realidade das demais regiões;

3) Em adultos, o uso desse método pode ser considerado uma alternativa viável para avaliar e monitorar o estado nutricional, em situações em que não é possível a medida direta dessas informações;

4) Em idosos, ainda não é possível recomendar a utilização das medidas auto-referidas por dois motivos: 1) os estudos não foram conduzidos especificamente com indivíduos acima de 60 anos; 2) esse grupo etário tende a estimar com menor acurácia essas medidas.

Sugere-se a realização de estudos nacionais com amostra representativa ou sub-amostra envolvendo crianças, adolescentes e idosos, com o intuito de verificar a validade das medidas antropométricas auto-referidas para avaliar o estado nutricional. Em adultos, recomenda-se a realização de pesquisas nas regiões Norte e Nordeste, uma vez que foi observada a inexistência de investigações nesses locais.

\section{REFERÊNCIAS BIBLIOGRÁFICAS}

1. Kuczmarski MF, Kuczmarski RJ. Nutritional assessment of older adults. In: Schlenker ED, editor, Nutrition in aging. 2nd ed, St Louis; Mosby-Year Book, 1993. p. 225-283.

2. Afonso FM, Sichieri R. Associação do índice de massa corporal e da relação cintura/quadril com hospitalizações em adultos do Município do Rio de Janeiro, RJ. Rev Bras Epidemiol 2002;5(2):153163.

3. Acuña K, Cruz T. Avaliação do estado nutricional de adultos e idosos e situação nutricional da população brasileira. Arq Bras Endocrinol Metab 2004;48(3):345-361.

4. Ell E, Camacho LA, Chor D. Perfil antropométrico de funcionários de banco estatal no Estado do Rio de Janeiro/Brasil: I- Índice de massa corporal e fatores sócio-demográficos. Cad Saúde Pública 1999;15(1):113-121.
5. Thommasen HV, Self B, Grigg A, Zhang W, Birmingham CL. The relationship between self-rated health, stress, health care, overall quality of life and weight in a rural population. Eat Weight Disord 2005;10(3):66-69.

6. Ministério da Saúde (BR). Vigilância de fatores de risco e proteção para doenças crônicas por inquérito telefônico (VIGITEL 2006): estimativas sobre freqüência e distribuição sócio-demográfica de fatores de risco e proteção para doenças crônicas nas capitais dos 26 Estados brasileiros e Distrito Federal em 2006. Brasília: Ministério da Saúde; 2007.

7. Ministério da Saúde (BR). Vigilância Alimentar e Nutricional (SISVAN): Orientações para a coleta e análise de dados antropométricos em serviços de saúde - Norma Técnica. Secretaria de Atenção à Saúde - Departamento de Atenção Básica Coordenação Geral da Política de Alimentação e Nutrição. Material preliminar; 2008.

8. Peixoto MRG, Benício MHA, Jardim PCBV. Validade do peso e da altura auto-referidos: o estudo de Goiânia. Rev Saúde Pública 2006;40(6):10651072.

9. Silveira EA, Araújo CL, Gigante DP, Barros AJD, Lima MS. Validação do peso e altura referidos para o diagnóstico do estado nutricional em uma população de adultos no Sul do Brasil. Cad Saúde Pública 2005;21(1):235-245.

10. Fonseca MJM, Faerstein E, Chor D, Lopes C. Validade de peso e estatura informados e índice de massa corporal: estudo pró-saúde. Rev Saúde Pública 2004;38(3)392-398.

11. Farias Jr. JC. Validade das medidas auto-referidas de peso e estatura para o diagnóstico do estado nutricional de adolescentes. Rev Bras Saúde Matern Infant 2007;7(2):167-174.

12. Tokmakidis SP, Christodoulos AD, Mantzouranis NI. Validity of self-reported anthropometric values used to assess body mass index and estimate obesity in Greek school children. J Adolesc Health 2007;40(4):305-310.

13. Lee K, Valeria B, Kochman C, Lenders CM. Self-assessment of height, weight, and sexual maturation: validity in overweight children and adolescents. J AdolesC Health 2006;39(3):346-352.

14. Galán I, Gandarillas A, Febrel C, Meseguer CM. Validación del peso y talla autodeclarados en población adolescente. Gac Sanit 2001;15(6):490-497.

15. Wang Z, Patterson CM, Hills AP. A comparison of self-reported and measured height, weight and BMI in Australian adolescents. Aust N Z Public Health 2002;26(5):473-478.

16. Abraham S, Luscombe G, Boyd C, Olesen I. Predictors of the accuracy of self-reported eight and weight in adolescent female school students. Int J Eat Disord 2004;36(1):76-82.

17. Rovira RF, Isabel, FP, Martinez MIM, Sanchez RR. Self-reported versus measured height, weight 
and body mass index in Spanish Mediterranean teenagers: effects of gender, age and weight on perceptual measures of body image. Ann Nutr Metab 2002;46(2):68-72.

18. Maranhão-Neto GA, Polito MD, Lira VA. Fidedignidade entre peso e estatura reportados e medidos e a influencia do histórico de atividade física em indivíduos que procuram a prática supervisionada de exercícios. Rev Bras Med Esporte 2005;11(2):141145.

19. Spencer EA, Appleby PN, Davey GK, Key TJ. Validity of self-reported height and weight in 4808 EPIC Oxford participants. Public Health Nutr 2002;5(4):561-565.

20. Payette H, Kergoat MJ, Shatenstein B, Boutier V, Nadon S. Validity of self-reported height and weight estimates in cognitively-intact and impaired elderly individuals. J Nutr Health Aging 2000;4(4):223-228.

21. Rowland ML. Self-reported weight and height. Am J Clin Nutr 1990;52(6):1125-1133.

22. Nieto-Garcia FJ, Bush TL, Keyl PM. Body mass definition of obesity: sensibility and specificity using self-reported weight and height. Epidemiology 1990;1(2):146-152.

23. Lawlor DA, Bedford C, Taylor M, Ebrahim S. Agreement between measured and self-reported weight in older women. Results from the British Women's Heart and Health Study. Age and Ageing 2002;31(3):169-174.
24. Kuczmarski MF, Kuczmarski RJ, Najjar M. Effects of age on validity of self-reported height weight, and body mass index: findings from of Third National Health and Nutrition Examination Survey, 19881994. J Am Diet Assoc 2001;101(1):28-34.

\section{Agradecimentos}

Os autores agradecem ao Prof. Ms. José Cazuza de Farias Júnior pelas contribuições e fornecimento de materiais para elaboração desse ponto de vista.

\section{Endereço para correspondência}

Raildo da Silva Coqueiro

Universidade Federal de Santa Catarina.

Núcleo de Pesquisa em Cineantropometria e Desempeno Humano (NuCIDH).

Campus Universitário - Trindade, Caixa

Postal 476

88040-900 - Florianópolis, SC.

E-mail: raildo@cds.ufsc.br 